
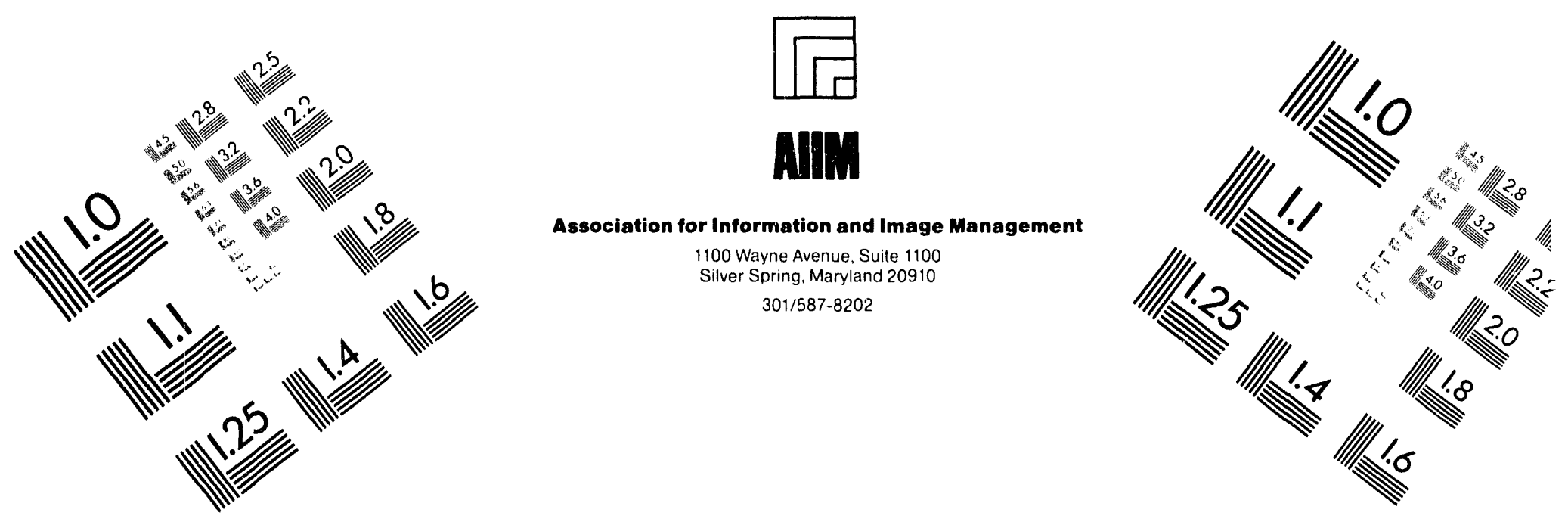

\title{
Centimeter
}

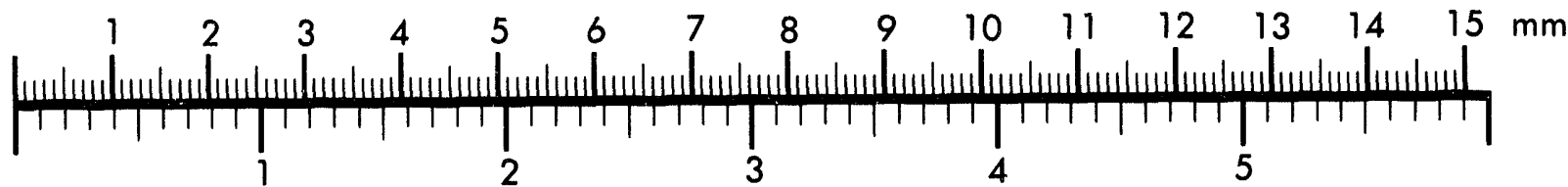

Inches
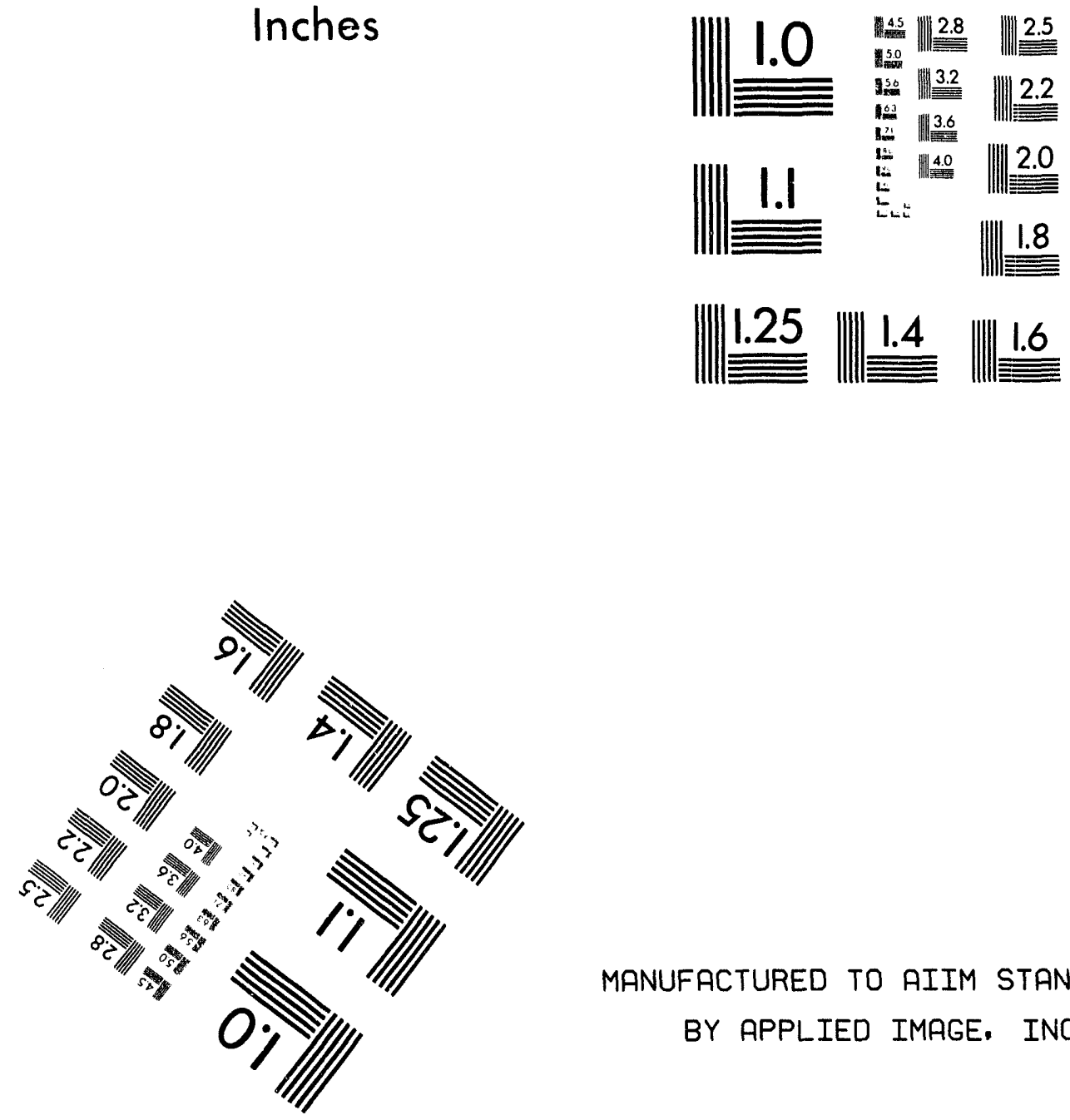

MANUFACTURED TO AIIM STANDARDS

BY APPLIED IMAGE, INC.

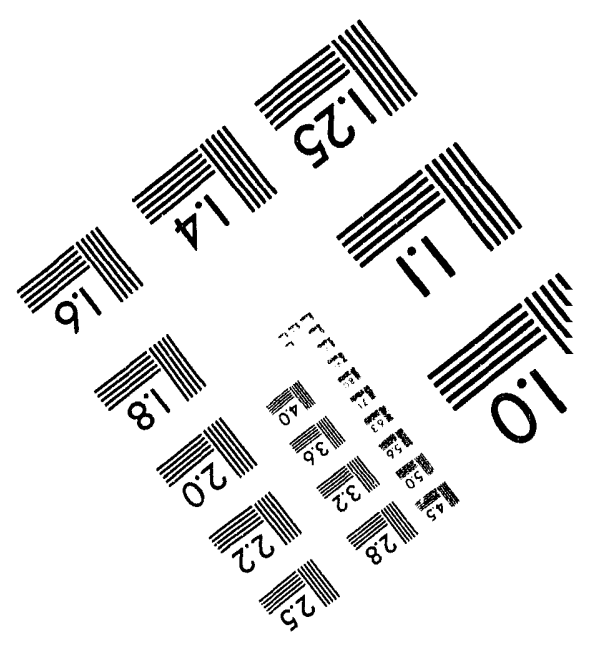



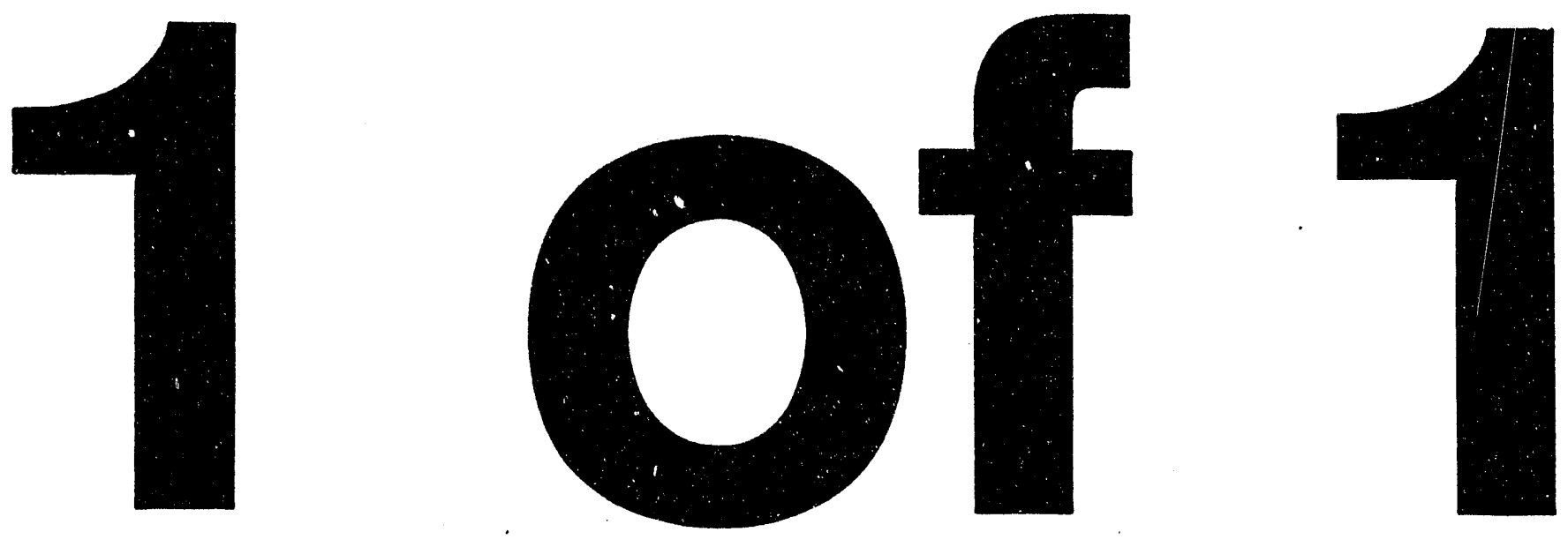
SAND94-0391

Distribution

Unlimited Release

Category UC-404

Printed April 1994

\title{
TIME-OF-FLIGHT DETECTOR FOR HEAVY ION BACKSCATTERING SPECTROMETRY
}

\author{
J. A. Knapp, J. C. Banks, and B. L. Doyle \\ Ion Solid Interactions and Defect Physics Department \\ Sandia National Laboratories \\ Albuquerque, New Mexico 87185
}

\begin{abstract}
This report describes the results of a two-year laboratory directed research and development project to explore advanced concepts in Heavy Ion Backscattering Spectrometry (HIBS), undertaken with the goal of extending the sensitivity of this relatively new technique to levels unattainable by any other existing trace element surface analysis. Improvements in sensitivity are required for the application of HIBS to contamination control in the microelectronics industry. Tools with sensitivity approaching $10^{8}$ atoms $/ \mathrm{cm}^{2}$ are expected to be essential for enabling advanced IC production by the year 2000. During the project we developed a new analysis chamber with channeling goniometer and a prototype time-of-flight detector with a demonstrated sensitivity of $\sim 5 \times 10^{8}$ atoms $/ \mathrm{cm}^{2}$ for $\mathrm{Au}$ on $\mathrm{Si}$ and $\sim 5 \times 10^{10}$ for $\mathrm{Fe}$, and sufficient mass resolution to separate contributions from $\mathrm{Fe}$ and $\mathrm{Cu}$.
\end{abstract}

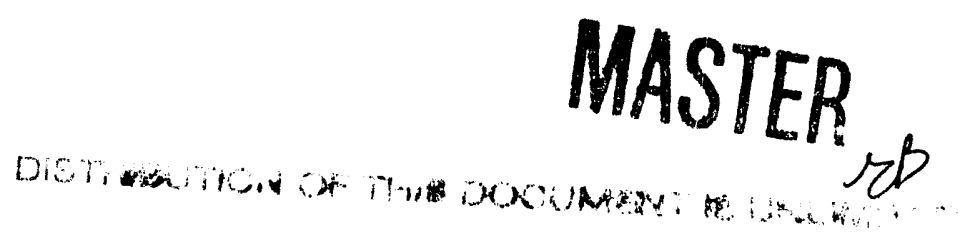




\section{TIME-OF-FLIGHT DETECTOR FOR HEAVY ION BACKSCATTERING SPECTROMETRY}

\section{Introduction}

For many new problems in materials research, particularly for contamination control in microelectronics, the sensitivity to surface impurities in surface analysis tools is inadequate. For example, by the turn of the century, very large scale integrated circuit processing will require contamination levels well below $1 \times 10^{9}$ atoms $/ \mathrm{cm}^{2}$ in both starting materials and introduced by processing.[1,2] The most sensitive of existing general-purpose tools, Total reflection X-Ray Fluorescence (TXRF), can detect $\sim 1 \times 10^{10}$ atoms $/ \mathrm{cm}^{2}$ levels of some elements such as $\mathrm{Fe}$ and $\mathrm{Cu}$, but for many others it is limited to $1 \times 10^{12}$ atoms $/ \mathrm{cm}^{2}$ or worse. Although a sensitivity of $10^{8}$ atoms $/ \mathrm{cm}^{2}$ is routinely claimed for TXRF, this is achieved only via pre-concentration through a technique called Vapor Phase Decomposition (VPD), wherein an acidic mist is applied to the wafer surface and then concentrated to a small spot.[3] Obviously, this technique has many uncertainties. HIBS provides a new ion beam analysis capability with the potential for providing the required sensitivity without pre-concentration for all elements above Ar. With pre-concentration the sensitivity would be approximately two orders of magnitude higher.

This project was undertaken to explore new concepts for HIBS analysis, in particular a time-offiight ion detector, and to optimize the design to achieve as high a sensitivity as possible. The result of the project was a unique Time-of-Flight (TOF) ion detector optimized for large solid angle. Two versions were constructed, the first fitting into ou: existing HIBS analysis chamber, with the second going into a new, larger chamber specifically built to accommodate it. The primary difference between the two was in the impedance matching of the electrical signal coming out of the multichannel plates; the second detector used $50 \mathrm{ohm}$ impedance matching collection cones, which take up considerably more room in the vacuum chamber. This second version has much better resistance to noise and solves problems with artifacts in the spectra which were due to electronic ringing in the first TOF detector. This change, together with other improvements in electronics and shielding of stray ions scattered around the chamber, have allowed us to achieve sensitivity of $\sim 5 \times 10^{8}$ atoms $/ \mathrm{cm}^{2}$ for Au on Si and $\sim 5 \times 10^{10}$ for Fe. These sensitivity numbers are for a single detector, whose design allows up to three detectors to be arrayed around the analysis beam to give correspondingly higher sensitivity. A HIBS system being built under a CRADA with SEMATECH will have such an arrangement of three parallel detectors, along with a larger beam spot, which also allows higher sensitivity. Another important parameter for detector perforrnance is the mass resolution, which for the new time-of-flight detector is now sufficient to separate $\mathrm{Fe}$ and $\mathrm{Cu}$. 


\section{Background}

When the possibilities for improved surface analysis techniques are considered, a good candidate, at least for heavy impurities on a light substrate, is a modification of Rutherford backscattering spectrometry (RBS) using low energy, heavy mass ion beams. RBS is typically performed using a 1-2 $\mathrm{MeV} \mathrm{He}^{+}$ion beam for the analysis, resulting in a sensitivity of $\sim 10^{13}$ atoms $/ \mathrm{cm}^{2}$ to impurities at or near the surface and is limited by system noise. In a backscattering experiment the number of particles scattered in the direction of the detector determines the yield of counts from that particular target, and for the pro; ser energy range is given by Rutherford's formula[4], as transformed from center-of-mass to a laboratory frame of reference differential scattering cross-section by Darwin[5]:

$\frac{d \sigma}{d \Omega}=\left(\frac{Z_{1} Z_{2} e^{2}}{2 E \sin ^{2} \theta}\right)^{2} \frac{\left\{\cos \theta+\left[1-\left(\frac{M_{1}}{M_{2}} \sin \theta\right)^{2}\right]^{\frac{1}{2}}\right\}^{2}}{\left[1-\left(\frac{M_{1}}{M_{2}} \sin \theta\right)^{2}\right]^{\frac{1}{2}}}$

where $Z_{1}$ and $Z_{2}$ are the atomic numbers of the projectile and target atom, $e$ is electron charge, and $E$ is the energy of the particle immediately before scattering. The average scattering cross section, $\sigma$, is defined as

$\sigma=\frac{1}{\Omega} \int \frac{d \sigma}{d \Omega} d \Omega$

where the integration is over $\Omega$, the finite solid angle spanned by the detector.

Examination of Eq. 1 will show that the backscattering yield is proportional to the square of the atomic number of the analyzing beam and inversely proportional to the square of its energy, so an enhanced yield can be obtained by using a higher $\mathrm{Z}$ ion beam at lower energy. Although this yield enhancement is well known, it is not widely exploited because pileup due to yield from the lower mass substrate overwhelms the signal from ions scattered by trace surface impurities. (Pileup occurs in an energy detector when multiple low-energy signals arrive simultaneously at a detector, such that they are indistinguishable from a single, higher-energy event). Pileup can be substantially reduced by a simple ranging foil in front of the detector which allows only particles backscattered from species heavier than the substrate to reach the detector.

Our original implementation of HIBS used a standard surface barrier particle energy detector (SBD) with a $C$ foil placed in front of the detector.[6,7] The primary advantages of the SBD approach are low cost and simplicity. Figure 1 shows a spectrum typical of our previous HIBS capability, obtained using a $300 \mathrm{keV} \mathrm{C}^{+}$beam from a sample deliberately contaminated with $\mathrm{Fe}$ and $\mathrm{Cu}$, as well as from a clean Si sample. The peak centered at channel 130 was from an unexpected contaminant. The inset shows a schematic of the experimental arrangement, which used up to three SBDs arranged in a circle around the incoming beam, each with $a \sim 5 \mu \mathrm{g} / \mathrm{cm}^{2}$ foil in front. The solid 
angle for each detector was $0.1 \mathrm{sr}$, and this spectrum was obtained with a single detector and $50 \mu \mathrm{C}$ total charge.

As can be seen from Fig. 1, a substantial background signal at low energy interferes with the analysis. This is partly due to electronic noise at the low signal levels involved, and partly due to backscattered particles from $\mathrm{Si}$ "leaking through" the foil. The latter is caused by energy straggling in the foil, in addition to whatever non-uniformity exists in these very thin foils. Where feasible, analyzing the sample with the incident beam along a channeling direction helps reduce this background; one of the improvements which was implemented during this project was a multisample goniometer to allow this. Clearly, for SBD-HIBS, the background is a problem for analysis: a tradeoff must be made between reducing the beam energy (to increase yield) and separating the signel from the low energy background. In practice, a lower limit of $250 \mathrm{keV}$ was found for SBDHIBs using a $\mathrm{C}^{+}$beam.

Further limitations in the SBD approach are inherent to the detectors themselves. The surface contact and underlying dead layer are in effect an additional foil thickness which must be taken into account (and which also, unlike the $\mathrm{C}$ foil, contributes to the electronic noise); special detectors

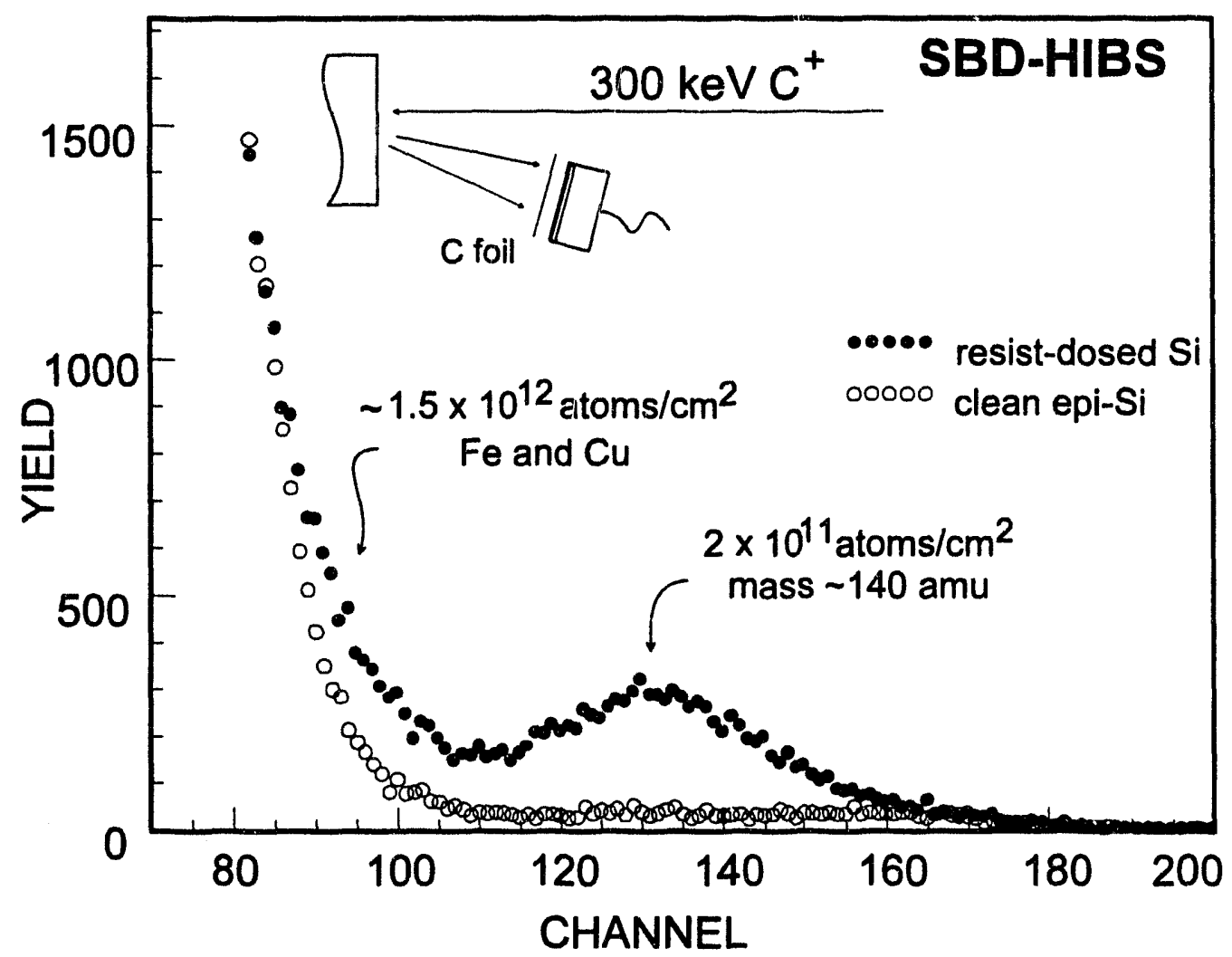

Figure 1. SBD-HIBS spectra obtained from a deliberately contaminated Si sample and from clean $\mathrm{Si}$, for comparison. The inset shows the experimental arrangement, with a surface barrier detector behind a $\mathrm{C}$ ranging foil. This is typical of the HIBS capability at the start of the project.

should be used with minimal surface dead layer thicknesses. Furthermore, the signal overlap with 
the low energy tail is exacerbated by the relatively poor energy resolution of the SBD in this application, which also leads to poor mass resolution. It is very difficult, for example, to separate contributions from $\mathrm{Fe}$ and $\mathrm{Cu}$, if both are present.

Figure 2 shows several TOF spectra typical of the new HIBS capability. $[9,10]$ These were taken during a study comparing different trace analysis techniques.[11] In this study, a series of samples were deliberately dosed with different levels of $\mathrm{Ni}$, using an ashed photoresist. The nominal doses associated with each spectrum: (a) $1 \times 10^{12} \mathrm{Ni} / \mathrm{cm}^{2}$, (b) $5 \times 10^{11} \mathrm{Ni} / \mathrm{cm}^{2}$, and (c) $1.5 \times 10^{11} \mathrm{Ni} / \mathrm{cm}^{2}$. The samples shown in (a) and (b) were prepared by a commercial vendor, while that shown in (c) was prepared at SEMATECH. HIBS analysis confirms the expected levels of $\mathrm{Ni}$ and also demonstrates that the samples were contaminated with $\mathrm{Br}$ and low levels of $\mathrm{Sn}$ and $\mathrm{Pb}$. The samples from the outside vendor all had substantial amounts of $\mathrm{Br}$, which was absent from the SEMATECH samples, while all samples exhibited $\mathrm{Sn}$ and $\mathrm{Pb}$. Although the samples were all examined with TXRF, these contaminants were missed, since TXRF is insensitive to these mass ranges. However, a follow-on study using VPD followed by ICP-MS showed that the low levels of $\mathrm{Sn}$ and $\mathrm{Pb}$ were indeed present on the wafers. This illustrates that even though the elemental identification capability of HIBS (i.e., mass resolution) is not as good as other techniques (e.g. TXRF), the lack of matrix effects and general sensitivity to all elements above Ar is very powerful. We will now describe the detector design.

The thrust of this project has been to explore alternative approaches to HIBS using a TOF detector, which gives substantially better energy resolution at the cost of greater complexity. TOF detection schemes for ion beam analyses have typically been optimized for good energy and mass resolution, with very small solid angles.[11] For the trace contamination analyses of HIBS, a larger solid angle is desirable, while energy resolution is not as critical. The advantages of TOF detection for this application include the ability to detect lower energy particles (with no dead layer in the detector), which in turns allows lower analysis energy and hence higher sensitivity. In addition, because the resulting spectrum is a time spectrum, the contributions from different masses are separated more widely at lower mass, which is the reverse of the situation for an energy spectrum. Thus the mass resolution is enhanced at lower mass, which is an advantage for application to problems of particular interest to the microelectronics community. The disadvantages of TOF ion detection are the greater complexity of the detector and its electronics, and the difficulty in achieving a large solid angle (needed for high sensitivity). The detector described in the rest of this report is the first TOF ion backscattering detector optimized for a relatively large solid angle. 


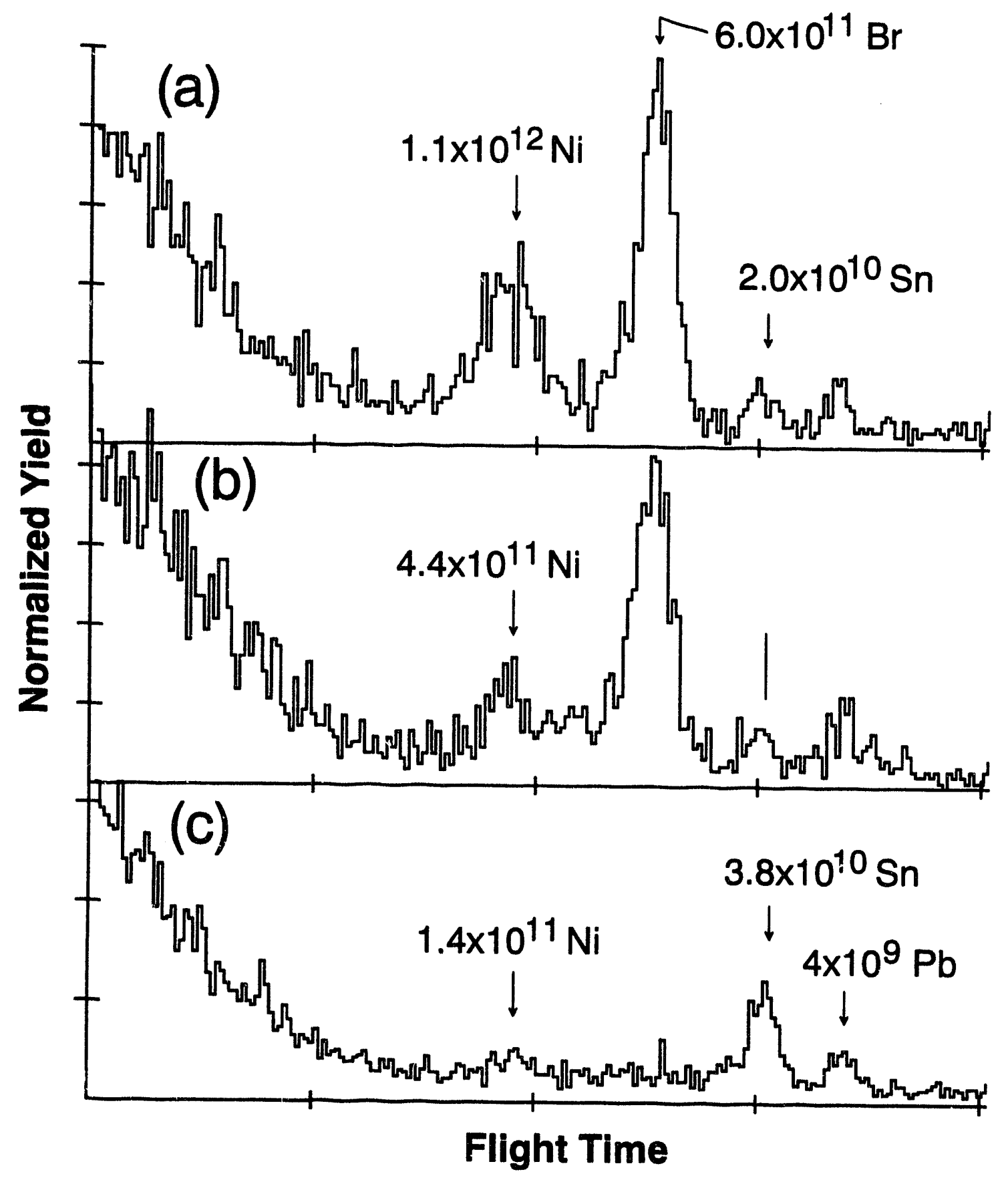

Figure 2. Three TOF-HIBS spectra obtained with $180 \mathrm{keV} \mathrm{N}^{+}$. Normalized yield is plotted versus flight time (decreasing from left to right). Each sample is Si dosed with Ni to nominal levels of (a) $1 \times 10^{12}$ atoms $/ \mathrm{cm}^{2}$, (b) $5 \times 10^{11}$ atoms $/ \mathrm{cm}^{2}$, and (c) $1.5 \times 10^{11}$ atoms $/ \mathrm{cm}^{2}$. The measured amount of $\mathrm{Ni}$ for each is shown, as well as $\mathrm{Br}, \mathrm{Sn}$ and $\mathrm{Pb}$ contamination peaks. These are typical of the new HIBS capability. 


\section{Time-of-Flight Detector}

Figure 3 shows a schematic of our TOF-HIBS detector, which uses a large area stop detector and a relatively short $12 \mathrm{~cm}$. flight path to maximize solid angle, while keeping energy resolution within acceptable bounds. As with the SBD approach to HIBS, a carbon foil is used to block signal from the substrate, but it now also provides an electron start pulse. The TOF detector system consists of two event timing detectors. As backscattered ions pass through the thin carbon foil, they produce secondary electrons which are accelerated through an adjacent grid and detected by an electron microchannel plate (MCP) assembly, producing a timing pulse. The ions continue their flight until stopped in the ion $\mathrm{MCP}$, giving another timing pulse.

Since it was desirable to maximize the solid angle for improving sensitivity, the angle subtended by the ion MCP is relatively large, which leads to kinematic broadening. That is, the kinematic factor (energy) for backscattering fron each mass changes substantially from one side of the plate to the other. The angles of both the foil and the ion MCP were chosen by computer simulation during the design phase to minimize this broadening. The foil and associated electron $\mathrm{MCP}$ are at $55^{\circ}$ relative to the sample plane, while the ion MCP is at $20^{\circ}$. The backscattering angie at the center of the ion $\mathrm{MCP}$ is $164^{\circ}$, so this plate is tilted $4^{\circ}$ away from being perpendicular to the average backscattered path. This optimization of the angles is the key to adapting the TOF approach to a large solid angle.

The ion beam is defined to $2 \mathrm{~mm}$ diameter with an aperture and then enters the chamber through a flight tube (not shown), leaving the tube a few $\mathrm{cm}$ before striking the sample. Both the aperture and flight tube are made from high purity aluminum. The samples are mounted on a 6 position, 2-axis

\section{Sandia Time-of-Flight Heavy Ion Backscattering Spectrometry (TOF-HIBS ) Prototype}

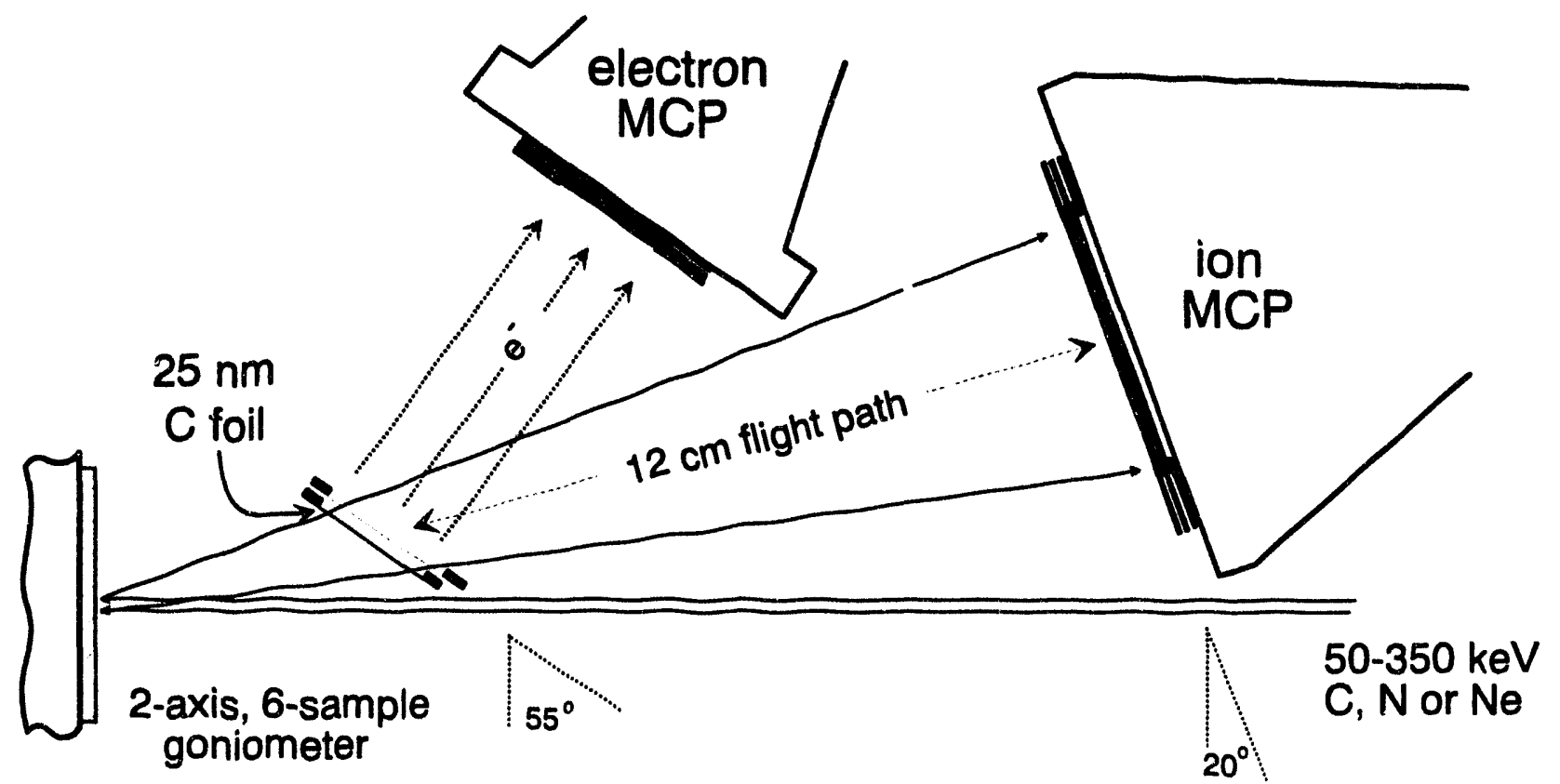

Figure 3. Schematic of the layout of the Time-of-Flight HIBS detector. 
goniometer, with only high purity $\mathrm{Al}$ or low $\mathrm{Z}$ material used for mounting. Backscattered particles pass through a $5 \mu \mathrm{g} / \mathrm{cm}^{2}$ carbon foil mounted on a foil assembly. This assembly consists of a rectangular foil frame, with a through center hole, and is covered on one side by a $90 \%$ transmission $\mathrm{Ni}$ mesh (Buckbee-Mears MN-17) to give support to the thin carbon foil. The mesh is spot welded to the stainless steel frame to ensure electrical contact and is tonded to the frame for strength, by a thin layer of epoxy (Varian Torr Seal) surrounding the through hole. The spectrographic grade carbon foil (Arizona Carbon Foil) is mounted to this assembly as per normal methods outlined in instructions from Arizona Carbon Foil. This assembly is then biased at $-800 \mathrm{~V}$ relative to ground. A second grid, at ground potential, is mounted $2.5 \mathrm{~mm}$ away at the back side of the foil. The foil serves two purposes: one, to range out the particles scattered from the light substrate, and second, to provide a pulse of electrons which is detected by the electron MCP assembly (Galileo ElectroOptics FTD-2003). Particles which exit the foil (in principle only those scattered from heavy impurities on the sample) pass along the $12 \mathrm{~cm}$ flight path and are detected by the ion MCP assembly (Galileo Electro-Optics FTD-4000). Both assemblies use chevron MCP arrangements (two microchannel plates mounted and oriented to reduce feedback and increase gain). The front plate of the ion MCP assembly has a $1260 \mathrm{~mm}^{2}$ active area.

This detector differs from our first prototype in several important ways. First, the microchannel plate assemblies have impedance matching conical anodes mounted on $50 \Omega$ BNC output connectors, for greatly improved resistance to system noise and ringing. Unfortunately, for the electron MCP the use of a BNC connector makes it more difficult to pass the signal through the
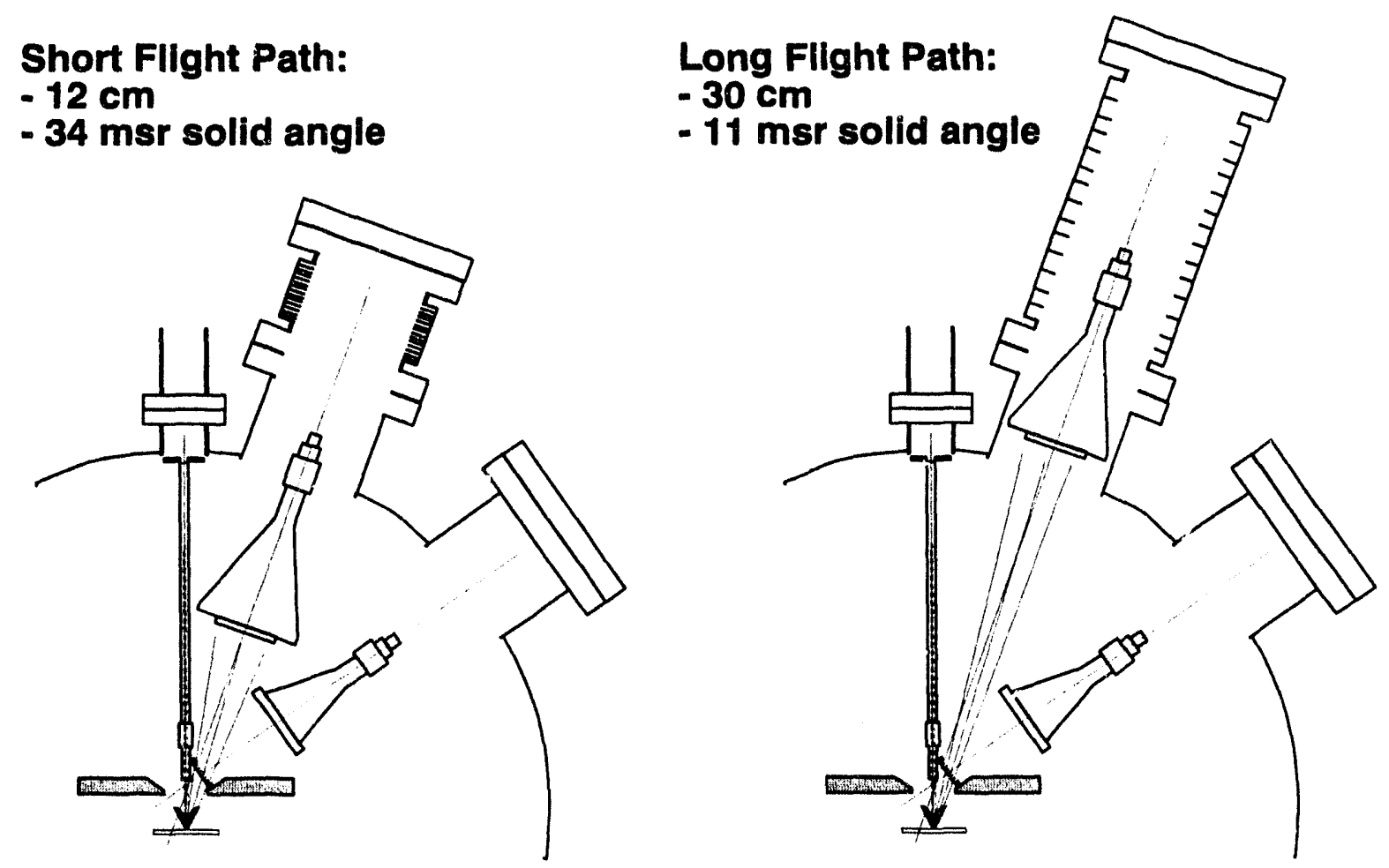

Figure 4. Schematic of the two configurations of the detector. The short flight path, high solid angle is for high sensitivity, the primary application; the long flight path configuration gives higher depth and mass resolution at the cost of sensitivity. 
chamber wall, since the front plate needs to be at ground, with the back plate, or signal at $+2000 \mathrm{~V}$. Standard $50 \Omega$ feedthroughs do not handle such high voltages. We took advantage of a decoupling system (Xenotech FTD-2003 Fast Pulse Decoupler) designed to solve this problem by Mendenhall and Weller in the course of their own research into the use of TOF detectors for low energy ion beam analysis.[11] The other major difference is that the ion MCP is mounted on a linear feedthrough such that it can be retracted $15 \mathrm{~cm}$ if desired, thereby doubling the flight path. This allows trading off sensitivity (solid angle) for somewhat improved mass resolution in specific experiments. Additionally, by changing to a lighter ion beam such as $\mathrm{He}$, the system can be used in this longer flight path mode for relatively high resolution depth profiling, rather than high sensitivity. The solid angle for the MCP in the short flight path is $34 \mathrm{msr}$, while for the long flight path it is $11 \mathrm{msr}$. Figure 4 shows a schematic of the two positions.

After the electrons and ions are detected by the MCPs, the signals are passed through a constant fraction discriminator (Ortec 935). The electron MCP signal is then delayed by $300-500 \mathrm{~ns}$, and the ion MCP pulse is used as the start pulse in a timc-to-amplitude converter (Ortec 567), with the delayed electron pulse serving for the stop pulse. This configuration increases the efficiency of the detector (since only counts that are significant start the timing sequence). The output of the time-toamplitude converter is fed to a standard PC-based pulse height analysis system for data collection.

\section{Sensitivity}

The sensitivity of HIBS can be increased in a number a ways: increasing the solid angle by using multiple detectors in parallel, using a larger beam spot, channeling the incident beam along a crystal axis of the substrate, or using a higher $Z$, lower-energy beam. On the other hand, several factors affect the ultimate sensitivity which can be achieved. The efficiency of the channelplates for

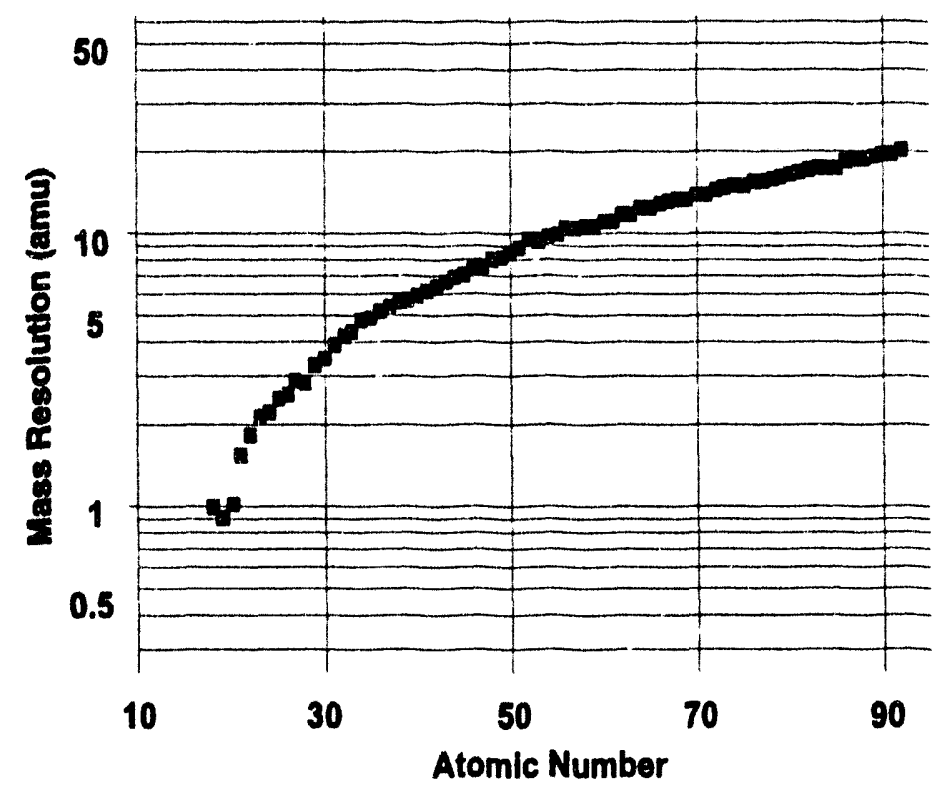

Figure 5. Approximate mass resolution vs. atomic number, in the short flight path configuration. detecting electrons, ions and neutrals in this energy range is not well known, nor is the electron-producing efficiency of the ranging foil. The channelplates also have a count rate limitation which may in turn limit the amount of beam current which can be used. Random coincidences (which have the same effect in a TOF detector as pulse pileup in an energy detector) are largely eliminated by the range-timing foil, but because of energy straggling and non-uniformities in this foil, some particles scattered from the substrate come through the foil, producing a background at 
lower flight time (see Fig. 2) and a small amount of random coincidences. Multiple scattering in the target, where particles undergo several small angle scattering events and exit the sample with higher energy than a single large-angle event would produce, is also expected to produce a background at very low levels [12] and may be part of the observed background.

Straggling in the foil is a more serious effect, dominating the observed time resolution and limiting the mass resolution that can be achieved. Furthermore, scattering in the foil results in some ions exiting at angles which don't intercept the ion channelplate, reducing the effective solid angle of the detector. This effect is stronger at lower energy, offsetting the gains that can be obtained by going to lower beam energy. Figure 5 shows the approximate mass resolution achieved with the short flight path using the $5 \mu \mathrm{g} / \mathrm{cm}^{2} \mathrm{C}$ foils.

Sputtering of the sample surface by the analysis beam is the ultimate limit to the statistics which can be obtained, and hence the sensitivity. The rate of sputtering will depend on the state of the impurity: particles will sputter differently than widely dispersed, low concentration layers, so it is difficult to predict the limit in advance. Experiments done during this project, wherein a sample was repeatedly analyzed on the same spot, demonstrated the rate of sputtering and suggested that the rate for a sub-monolayer impurity is lower than predicted by a simple sputtering model using bulk sputtering rates.

\section{Detection Limits on Si - HIBS vs. TXRF}

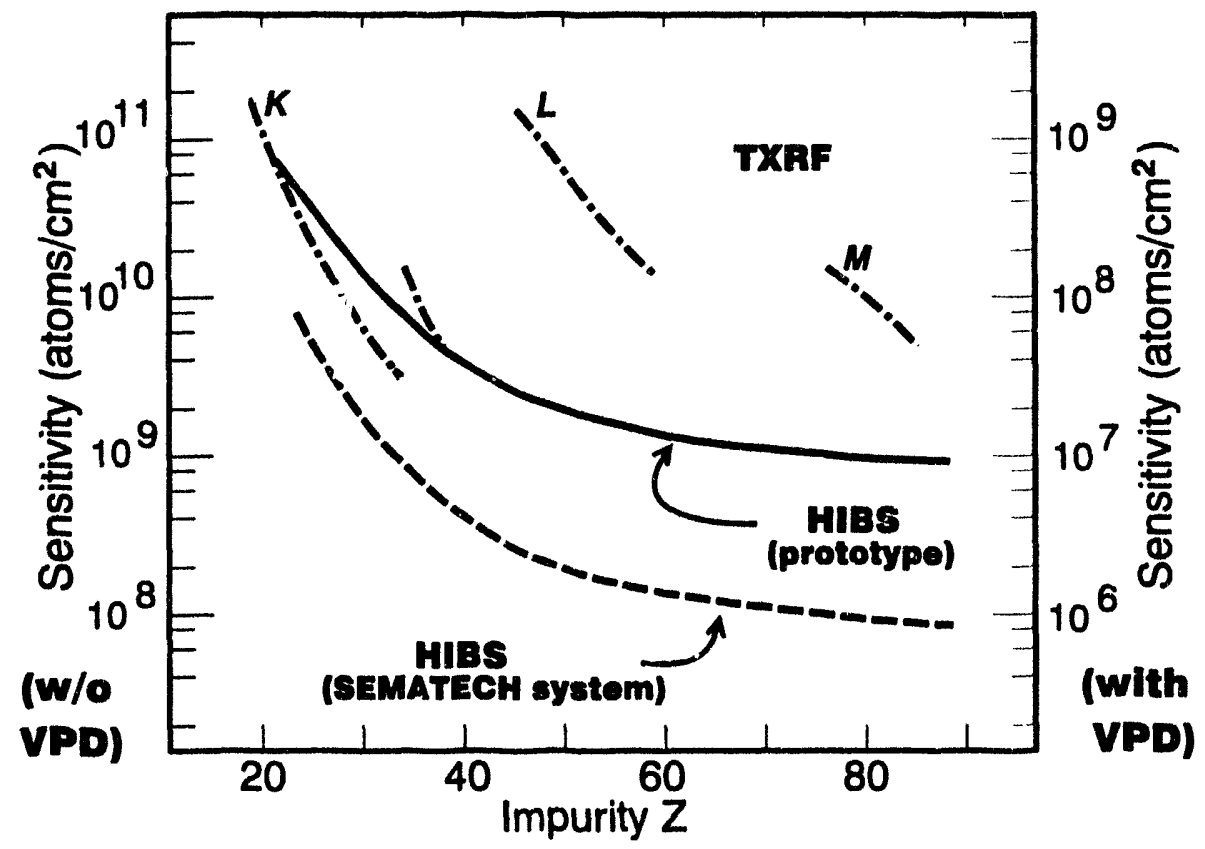

Figure 6. Detection limits for contaminants on Si. The solid line is the demonstrated sensitivity for the research HIBS prototype, while the dashed line is the anticipated sensitivity for the SEMATECH system, with three parallel detectors and a larger beam spot. Approximate sensitivities for TXRF are also plotted for comparison. 
The sensitivity of the system in its present, single detector configuration ranges from $\sim j \times 10^{10}$ atoms $/ \mathrm{cm}^{2}$ for $\mathrm{Fe}$ to $\sim 5 \times 10^{8}$ atoms $/ \mathrm{cm}^{2}$ for $\mathrm{Au}$, both on a Si substrate. Figure 6 compares the sensitivity of the prototype to that of TXRF, along with the expected sensitivity of a HIBS prototype we are constructing under a CRADA with SEMATECH. The latter system, with three parallel detectors, optimized foils and a larger beam spot, should reach sensitivities of $5 \times 10^{9}$ atoms $/ \mathrm{cm}^{2}$ for $\mathrm{Fe}$ and $1 \times 10^{3}$ atoms $/ \mathrm{cm}^{2}$ for $\mathrm{Au}$. The figure shows sensitivity scales with and without pre-concentration by VPD.

\section{Acknowledgments}

Technical assistance by D. L. Buller and K. G. Minor is gratefully acknowledged. The collaborations of C. P. Resnick of Sandia Labs and A. C. Diebold of SEMATECH in studies of contamination of Si has been essential. This work was performed at Sandia National Laboratories and supported by the U.S. Department of Energy under contract no. DE-AC04-94AL85000. 


\section{References}

[1] See for example, Proceedings of Microcontamination 91, Oct. 16-18, 1991 (unpublished).

[2] Alain C. Diebold, P. Maillot, M. Gordon, J. Baylis, J. Chacon, R. Witowski, H. F. Arlinghaus, J. A. Knapp, and B. L. Doyle, J. Vac. Sci. Technol. A 10(4) (1992) 2945.

[3] M. Meuris, M. Heyns, W. Kuper, S. Verhaverbeke, and A. Philipossian, ECS, Washington, D. C., May 1991 (unpublished).

[4] E. Rutherford, Phil. Mag. 21 (1911) 669.

[5] C. G. Darwin, Phil. Mag. 28 (1914) 499.

[6] B. L. Doyle, J. A. Knapp, and D. L. Buller, Nucl. Inst. and Meth. B42 (1989) 295.

[7] J. A. Knapp and B. L. Doyle, Nucl. Inst. and Meth. B45 (1990) 143.

[8] J. A. Knapp and J. C. Banks, Nucl. Inst. and Meth. B79 (1993) 457.

[9] J. A. Knapp, J. C. Banks, and B. L. Doyle, Nucl. Inst. and Meth., in press.

[10] W. F. Calaway, S. R. Coon, M. J. Pellin, D. M. Gruen, M. Gordon, A. C. Diebold, P. Maillot, J. C. Banks, ard J. A. Knapp, Surface and Interface Analysis (in press).

[11] M. H. Mendenhall and R. A. Weller, Nuc. Inst. and Meth. B47 (1990) 193.

[12] D. K. Brice, Nucl. Instr. and Meth. B69 (1992) 349. 


\section{DISTRIBUTION:}

1 SEMATECH

Attn: Alain C. Diebold, 2706 Montopolis Dr.

Austin TX 78741

3 Vanderbilt University

Attn: Robert A. Weller Marcus Mendenhall Dianne Pederson

Box 1687 Station B

Nashville, TN 37235

1 Advanced Micro Devices

Attn: Erjan Adem

901 Thompson Place

M/S 178

Sunnyvale CA 94086

1 Motorola, Inc.

Attn: Rich Gregory

2200 W. Broadway Rd.

M/S M360

Mesa AZ 85202

$1 \quad$ AT\&T Bell Labs

Attn: Dale Jacobson

Rm 1E-365

600 Mountain Ave.

Murray Hill NJ 07974-0636

2 Texas Instruments, Inc.

Attn: Mark Anthony Joe Keenan

Research Bldg. East, M/S 147

13588 N. Central Expressway

Dallas TX 75243
2 Advanced Micro Devices

Attn: John Lowell, M/S 547

Don Tiffin, M/S 554

5204 E. Ben White Blvd.

Austin TX 78741

1 Hewlett Packard Co.

Attn: Chris Nauka

3500 Deer Park Rd.

Palo Alto CA 94304

1 Digital Equipment Corp.

Attn: Howard Smith

75 Reed Road

M/S HI,02-3/M09

Hudson MA 01749

2

1

1

50

2

1

5

G. D. Tipton, 1332
MS 1083 C. P. Resnick, 1332

1078 C. W. Gwyn, 1302

J. D. McBrayer, 1302

1082 R. S. Blewer, 1305

0368 C. Adkins, 1815

1056 J. A. Knapp, 1111 (25)

J. C. Banks, 1111 (20)

B. L. Doyle, 1111 (5)

0320 Laura Lopez, 1011

9018 Central Technical Files, 8523-2

0899 Technical Library, 7141

0619 Technical Publications, 7151

1119 Document Processing, 7613-2

For DOE/OSTI 

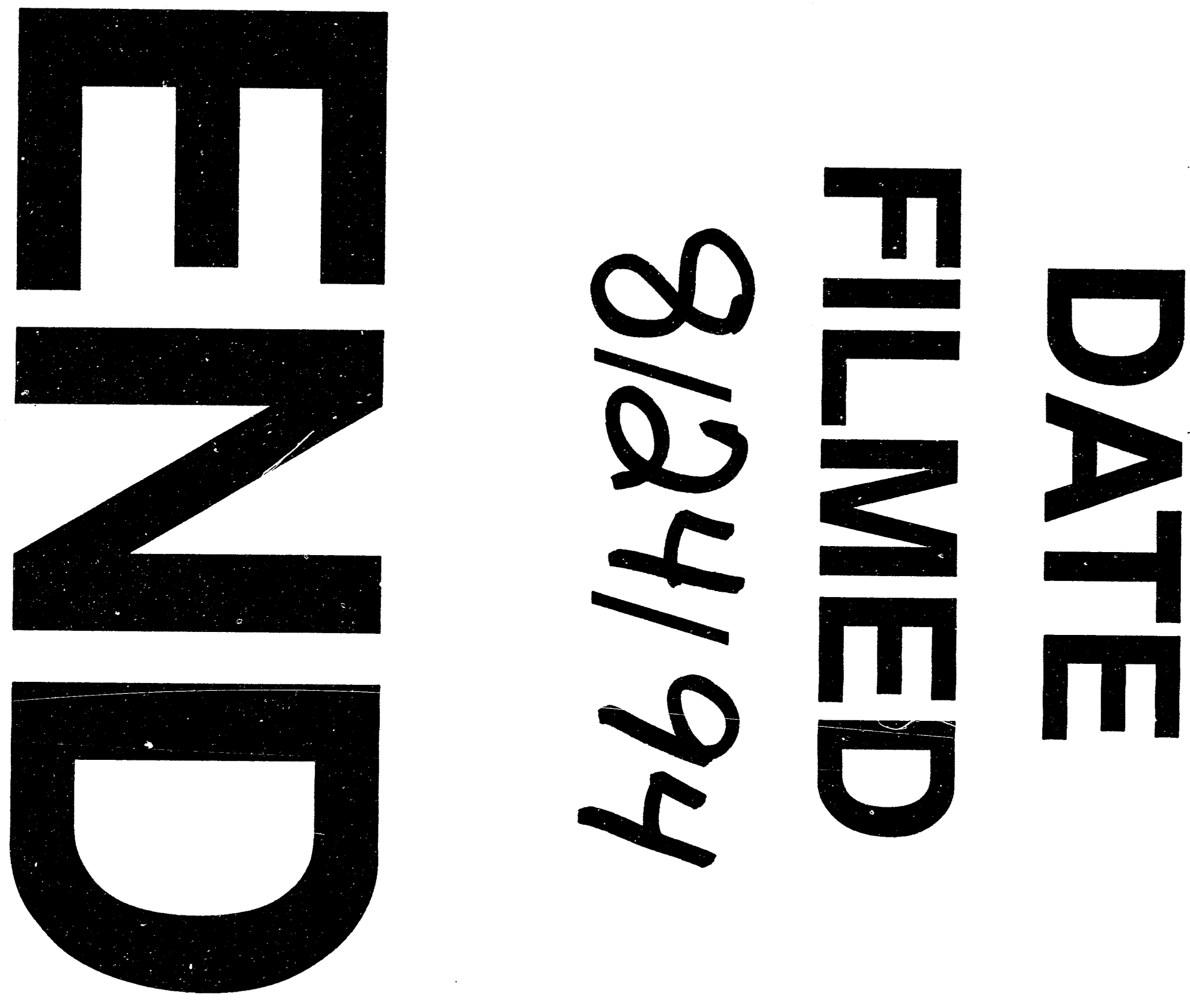
